\title{
Profile of neuromyelitis optica and spectrum disorders in an Indian cohort
}

Neuromyelitis optica (NMO) or Devic's syndrome, initially considered a severe variant of multiple sclerosis (MS), has been identified as a distinct autoimmune disease in recent decades. NMO peculiar clinical features, consisting of the combination of monophasic or recurrent episodes of optic neuritis $(\mathrm{ON})$ and myelitis (index events) without evidence of clinical disease outside of optic-spinal regions, ${ }^{[1]}$ contributed to its new nosological definition. In 2004, aquaporin-4-specific water channel antibodies or NMOIgG were discovered and they were found to be a highly specific and moderately sensitive biomarker for NMO ${ }^{[2]}$ In fact, the 2006 revised NMO criteria introduced, in addition to the index events, the presence of at least 2 of 3 supportive criteria: Magnetic resonance imaging (MRI) evidence of longitudinally extensive transverse myelitis (LETM) (at least 3 vertebral segments); brain MRI nondiagnostic for MS; and NMO-IgG seropositivity. ${ }^{[3]}$ These criteria led to the definition of NMO spectrum disorders (NMOSD), which includes conditions with both NMO-IgG seropositivity and one of the index events, ${ }^{[3]}$ embracing cases with symptoms other than optic-spinal involvement.

Neuromyelitis optica is a severe and invalidating disease, more frequent in non-Caucasian, especially Asian, countries although it has been described in all continents and races. In comparison to MS, NMO is associated with a greater imbalance between women and men, a higher median age of onset (35-45 years) and a lower prevalence (1-4.4/100.000). ${ }^{[4]}$ An early differential diagnosis of $\mathrm{NMO} / \mathrm{NMOSD}$ from MS is particularly important due to the necessity of rapid introduction of immunosuppressive therapy, since MS disease-modifying drugs may exacerbate these disorders. ${ }^{[5]}$

There are few epidemiological, clinical and radiological data on NMO/NMOSD in India. ${ }^{[6,7]}$ Indeed, larger studies

\begin{tabular}{|l|l|}
\hline \multicolumn{2}{|c|}{ Access this article online } \\
\hline Quick Response Code: & Website: \\
\hline & www.ruralneuropractice.com \\
\cline { 2 - 3 } & \\
\hline$\square$ & \\
\hline
\end{tabular}

are needed in order to have a deep knowledge of these diseases, thus favoring an early diagnosis and appropriate treatment. These data will be particularly important since $\mathrm{NMO} / \mathrm{NMOSD}$ accounts for up the $13.9 \%$ of inflammatory demyelinating disorders of the central nervous system in Indian population, with a prevalence rate of $2.6 / 100,000{ }^{[6]}$

In this new retrospective study, ${ }^{[8]}$ the authors describe epidemiological characteristics, clinical presentations, recurrence rate, treatment and response to therapy in 21 $\mathrm{NMO}$ and $6 \mathrm{NMOSD}$ patients among Indian population. Although the authors did not report NMO/NMOSD prevalence rate in their population, patients' sample size is quite large and the mean follow-up period is rather long (5 years).

In line with previous studies, the authors found that a recurrent disease course is more common than monophasic illness (73\% vs. $27 \%)$. Among 83 recurrent events, ON was found in $23 \%$, LETM in $56.62 \%$, both ON and LETM in $16.9 \%$ of polyphasic patients, being these data similar to those reported in a recent work on an Indian NMO/ NMOSD cohort. ${ }^{[7]}$ Even the median number of relapses was similar in both studies: 4 (range: $2-8$ ) during a mean follow-up of 5 years in the former, ${ }^{[8]}$ and 3 (range: $1-15$ ) during a median follow-up of 3 years (range: $0.33-30$ years) in the latter. ${ }^{[7]}$ Moreover, the mean age of symptom onset of 27 years (range: 9-58) found in this study is similar to that median of 26.5 (range: 8-72) of a previous Indian study, ${ }^{[7]}$ but it is lower than that reported in previous studies performed in American and Western countries, ${ }^{[1,4]}$ suggesting that $\mathrm{NMO} / \mathrm{NMOSD}$ cases in Indian population might tend to present at an earlier age.

The authors confirmed a strong female preponderance for NMO/NMOSD. ${ }^{[8]}$ However, women/men ratio in this study was lower compared to a recent paper on the Indian $\mathrm{NMO} / \mathrm{NMOSD}$ cohort, ${ }^{\left[{ }^{[]}\right]}$(female: male $=4.2: 1$ and $7.8: 1$, respectively), but higher in the monophasic than in the polyphasic form (6:1 and $3.75: 1$, respectively) ${ }^{[8]}$ in contrast to previous literature data. ${ }^{[1]}$

A limitation of this study is that only the $50 \%$ of patients were tested for serum NMO-IgG and the authors did not 
clarify if NMO-IgG testing were performed at the time of a relapse or at variable intervals after a relapse. Among NMO-IgG tested patients only the $61 \%$ were found to be seropositive, in contrast to a higher percentage reaching the $84 \%$ reported in another Indian cohort. ${ }^{[7]}$ Cerebrospinal fluid pleocytosis was noted in $33 \%$ patients with a mean of 11 leukocytes (range: $2-75 / \mathrm{mm}^{3}$ ) and protein levels ranged $18-70 \mathrm{mg} \%,{ }^{[8]}$ whereas the previous study found a median value of 10 leukocytes (range: $2-940 / \mathrm{mm}^{3}$ ) with a median protein level of 40.5 (range: $16-790 \mathrm{mg} \%$ ) including likely more severe cases. ${ }^{[7]}$

Only the $50 \%$ of patients received disease-modifying drugs (mostly azathioprine, but also methotrexate, mitoxantrone, monthly intravenous methylprednisolone).$^{[8]}$ The 2010 EFNS guidelines for NMO treatment considered azathioprine associated initially with prednisolone, and rituximab as the first line treatments while cyclophosphamide, mitoxantrone, mycophenolate and methotrexate as second line therapies. ${ }^{[5]}$ Interestingly, this study reported a good outcome during the long follow-up period with no direct NMO related mortality. Such data are not in line with the literature data showing a generally poor prognosis, ${ }^{[1,4]}$ and might be explained by probable inclusion of more benign NMO cases in this study.

Tatiana Koudriavtseva, Rosaria Renna

Department of Neurosciences, Multiple Sclerosis Center, Unit of Neurology, Regina Elena National Cancer Institute, Rome, Italy
Address for correspondence: Dr. Tatiana Koudriavtseva, Department of Neurosciences, Multiple Sclerosis Center, Unit of Neurology, Regina Elena National Cancer Institute, Via Elio Chianesi 53, 00144 - Rome, Italy

E-mail: tatiana.koud@gmail.com

\section{References}

1. Wingerchuk DM, Hogancamp WF, O’Brien PC, Weinshenker BG. The clinical course of neuromyelitis optica (Devic's syndrome). Neurology 1999;53:1107-14.

2. Lennon VA, Wingerchuk DM, Kryzer TJ, Pittock SJ, Lucchinetti CF, Fujihara $\mathrm{K}$, et al. A serum autoantibody marker of neuromyelitis optica: Distinction from multiple sclerosis. Lancet 2004;364:2106-12.

3. Wingerchuk DM, Lennon VA, Pittock SJ, Lucchinetti CF, Weinshenker BG. Revised diagnostic criteria for neuromyelitis optica. Neurology 2006;66:1485-9.

4. Drori T, Chapman J. Diagnosis and classification of neuromyelitis optica (Devic's syndrome). Autoimmun Rev 2014;13:531-3.

5. Sellner J, Boggild M, Clanet M, Hintzen RQ, Illes Z, Montalban X, et al. EFNS guidelines on diagnosis and management of neuromyelitis optica. Eur J Neurol 2010;17:1019-32.

6. Pandit L, Kundapur R. Prevalence and patterns of demyelinating central nervous system disorders in urban Mangalore, South India. Mult Scler 2014;20:1651-3.

7. Barhate KS, Ganeshan M, Singhal BS. A clinical and radiological profile of neuromyelitis optica and spectrum disorders in an Indian cohort. Ann Indian Acad Neurol 2014;17:77-81.

8. Jagtap SA, Mandliya A, Sarada C, Nair MD. Neuromyelitis optica (NMO) and NMO spectrum disorder: Natural history and long term outcome, An Indian experience. J Neurosci Rural Pract 2015;6:331-5.

How to cite this article: Koudriavtseva T, Renna R. Profile of neuromyelitis optica and spectrum disorders in an Indian cohort. J Neurosci Rural Pract 2015;6:295-6.

Source of Support: Nil. Conflict of Interest: None declared. 